\title{
Doença veno-linfática: alterações linfocintilográficas nas úlceras venosas
}

\author{
Venous-lymphatic disease: lymphoscintigraphic abnormalities in \\ venous ulcers
}

\author{
José Humberto Silva ${ }^{1}$, Maria del Carmen Janeiro Perez ${ }^{1}$, Newton de Barros Jr. ${ }^{1}$, \\ Mário Luiz Vieira Castiglioni ${ }^{2}$, Neil Ferreira Novo ${ }^{3}$, Fausto Miranda Jr. ${ }^{1}$
}

\section{Resumo}

Contexto: O sistema linfático tem papel relevante em qualquer tipo de edema periférico. Atualmente, a linfocintilografia é considerada o principal exame para diagnóstico da doença linfática das extremidades. Embora haja associação entre edema linfático e úlcera de estase venosa crônica, a fisiopatologia dessas alterações permanece indefinida.

Objetivo: Verificar as alterações linfocintilográficas qualitativas que ocorrem em pacientes portadores de úlceras de estase dos membros inferiores.

Métodos: Quarenta pacientes portadores de úlcera de estase venosa crônica ou cicatriz unilateral foram submetidos a linfocintilografia de ambos os membros inferiores. Foram estudados 25 mulheres e 15 homens, com média de idade de 53,7 anos ( 28 a 79 anos) e tempo médio de úlcera de 71,5 meses ( 3 a 240 meses). Foram comparados qualitativamente os parâmetros linfocintilográficos dos membros inferiores, previamente classificados em três grupos de acordo com a classificação clínica, etiológica, anatômica e patológica (CEAP): I, membros sem sinais clínicos de doença venosa ou com telangiectasias e veias reticulares (classes 0 e 1); II, membros inferiores com veias varicosas, edema e/ou alterações de pele e subcutâneo (classes 2, 3 e 4); III, membros inferiores com úlcera e/ou cicatriz (classes 5 e 6 ).

Resultados: Quando foi comparada a presença de alterações linfocintilográficas dos membros com úlcera ou cicatriz (grupo III classes 5 e 6) com as dos membros sem úlcera (grupos I e II - classes 0 , $1,2,3$ e 4), houve diferença significativa $(\mathrm{p}<0,001)$. Estratificando os membros inferiores de acordo com a classificação CEAP, também foi observada diferença estatisticamente significante $(p<0,001)$, sendo as alterações linfocintilográficas presentes em $72,5 \%$ no grupo III (classes 5 e 6), 30,8\% no grupo II (classes 2,3 e 4 ) e $7,1 \%$ no grupo III (classes 0 e 1). Em relação aos parâmetros analisados na linfocintilografia, ocorreu diferença significativa entre o grupo III e os outros grupos quanto à retenção de radiofármaco, adenomegalia inguinal e refluxo dérmico. Não houve significância nos parâmetros linfonodo poplíteo e circulação colateral.

Conclusão: Considerando os resultados, conclui-se que quanto mais grave a estase venosa crônica, maiores serão as alterações linfocintilográficas observadas, corroborando a associação entre doença venosa e linfática e entre linfedema secundário e estase venosa crônica.

Palavras-chave: Úlcera, estase venosa, linfocintilografia.

\begin{abstract}
Background: The lymphatic system plays a relevant role in any type of peripheral edema. Lymphoscintigraphy is currently considered the primary test in the diagnosis of lymphatic disease of the lower limbs. Although there is an association between lymphatic edema and chronic venous ulcers, the physiopathology of such changes remains uncertain.
\end{abstract}

Objective: To assess qualitative lymphoscintigraphic findings in patients with chronic venous ulcers of the lower limbs.

Methods: Forty patients with unilateral chronic venous ulcer or scar were submitted to bilateral lymphoscintigraphy of the lower limbs. The sample was comprised of 25 women and 15 men, with a mean age of 53.7 years ( 28 to 79 ) and mean ulcer duration of 71.5 months ( 3 to 240 months). Lymphoscintigraphic parameters were qualitatively compared among three groups of lower limbs previously classified according to the clinical, etiologic, anatomic and pathologic classification (CEAP): I, limbs without clinical signs of venous disease or with telangiectasias and/or reticular veins (classes 0 and 1); II, limbs with varicose veins, edema and/or skin and subcutaneous alterations (classes 2, 3 and 4); III, lower limbs with ulcer and/or ulcer scars (classes 5 and 6$)$.

Results: There was a significant difference $(p<0.001)$ in the comparison of lymphoscintigraphic findings of the lower limbs with (group III - classes 5 and 6) and without ulcers/scars (groups I and II - classes $0,1,2,3$ and 4). There was also a significant difference ( $p<$ 0.001 ) in the comparison of groups according to the clinical CEAP classification: lymphoscintigraphic abnormalities were present in $72.5 \%$ in group III (classes 5 and 6), in 30.8\% in group II (classes 2, 3 and 4), and in $7.1 \%$ in group I (classes 0 and 1). There was a statistically significant difference between group III and the other groups with regard to radiotracer retention, inguinal adenomegaly and dermal reflux. There was no significance as to the parameters popliteal lymph node and collateral circulation.

Conclusion: The more severe the venous chronic stasis, the more lymphoscintigraphic abnormalities were observed, corroborating the association between venous and lymphatic disease and between chronic venous stasis and secondary lymphedema.

Keywords: Ulcer, venous stasis, lymphoscintigraphy.

1. Disciplina de Cirurgia Vascular, Departamento de Cirurgia, Universidade Federal de São Paulo - Escola Paulista de Medicina (UNIFESP-EPM), São Paulo, SP.

2. Setor de Radioisótopos, Departamento de Diagnóstico por Imagem, UNIFESP-EPM, São Paulo, SP.

3. Professor titular, Saúde Pública, Universidade de Santo Amaro (UNISA), Santo Amaro, SP.

Não foram declarados conflitos de interesse associados à publicação deste artigo.

Artigo submetido em 27.08.08, aceito em 15.11.08.

J Vasc Bras. 2009;8(1):33-42.

Copyright $@ 2009$ by Sociedade Brasileira de Angiologia e de Cirurgia Vascular 


\section{Introdução}

A insuficiência venosa crônica é conceituada como as alterações decorrentes da hipertensão venosa de longa duração, que ocorrem em pele e tecido celular subcutâneo por insuficiência valvular e/ou por obstrução venosa. A obstrução e/ou insuficiência valvular venosa leva ao aumento de pressão venosa em veias, vênulas e capilares venosos (por aumento da pressão hidrostática e da permeabilidade), distensão da parede capilar e aumento dos poros interendoteliais, causando acúmulo de proteínas de maior peso molecular no tecido e aumento da pressão osmótica tissular ${ }^{1}$.

O sistema linfático tem papel importante em qualquer tipo de edema periférico ${ }^{2}$, havendo estreita relação anatômica entre veias e linfáticos superficiais. $\mathrm{Na}$ insuficiência venosa crônica, pode ocorrer insuficiência secundária da drenagem linfática, por sobrecarga volumétrica em linfáticos inicialmente normais, que multiplicam sua função para compensar o edema decorrente da estase venosa. No início do processo, há aumento do fluxo linfático para retirada do excesso de proteínas e fluido local. Quando a oferta excede a capacidade dos linfáticos, o aumento de proteínas de alto peso molecular e de líquido no tecido favorece os quadros de linfangites e erisipelas, que podem causar trombose dos canalículos linfáticos e consequente piora progressiva do edema e das demais complicações, como a lipodermatoesclerose e úlcera de estase. Quando o paciente apresenta linfedema secundário à estase venosa crônica, considera-se que o paciente apresenta uma doença veno-linfática ${ }^{3}$.

A linfocintilografia é considerada, nos dias de hoje, o principal exame para o diagnóstico da doença linfática das extremidades, atingindo sensibilidade de cerca de $90 \%$ e especificidade de $100 \%{ }^{4,5}$. Entretanto, são encontrados poucos trabalhos na literatura relacionando úlcera de estase com lesão linfática, utilizando a linfocintilografia ${ }^{6,7}$.

A falta de padronização ou a não-especificação dos parâmetros analisados e a controvérsia quanto aos resultados desses estudos levaram à avaliação qualitativa das alterações linfocintilográficas presentes em portadores de úlcera de estase venosa crônica unilateral, aberta ou cicatrizada, em membros inferiores, comparando-as com possíveis alterações encontradas nos membros contralaterais sem úlcera. Além disso, outro objetivo deste estudo foi verificar a correlação entre as alterações encontradas no exame linfocintilográfico e a gravidade da estase venosa clinicamente classificada de acordo com a CEAP ${ }^{8}$.

\section{Métodos}

Foram avaliados prospectivamente 40 pacientes recrutados no ambulatório da disciplina de cirurgia vascular da UNIFESP-EPM, com diagnóstico de úlcera de estase atual ou cicatrizada em apenas um dos membros inferiores, no período de novembro de 2002 a dezembro de 2003. O projeto foi analisado e aprovado pelo Comitê de Ética em Pesquisa UNIFESP-EPM (n ${ }^{\circ}$ 1002/02) e todos os pacientes assinaram o Termo de Consentimento Livre e Esclarecido.

A população estudada era composta de 25 mulheres e 15 homens, com média de idade de 53,7 anos (28 a 79 anos). Todos eram portadores de úlcera de estase venosa atual ou cicatrizada, unilateralmente, com média de duração das úlceras de 71,4 meses (3 a 240 meses). Foram excluídos os pacientes portadores de úlcera bilateral, para que os membros pudessem ser comparados quanto às alterações linfocintilográficas e também pacientes com úlceras apresentando sinais de infecção. Tais pacientes foram submetidos a exame clínico, agrupados de acordo com a classificação CEAP e estudados com linfocintilografia bilateral dos membros inferiores.

No grupo I, foram englobadas as classes 0 e 1 , com membros sem sinais clínicos de doença venosa ou com teleangiectasias e/ou veias reticulares. No grupo II, foram englobadas as classes 2,3 e 4, com portadores de veias varicosas, edema e/ou alterações de pele e subcutâneo. E no grupo III, foram englobadas as classes 5 e 6 , com alterações das classes anteriores e com úlcera aberta ou cicatrizada.

A linfocintilografia foi realizada com injeção intradérmica de $185 \mathrm{MBq}(5 \mathrm{mCi})$ de Dextran tecnécio ${ }^{99 \mathrm{~m}}$, no $1^{\circ}$ espaço interdigital, bilateralmente. As aquisições de imagens foram realizadas nos tempos 1 e 3 horas após a injeção ${ }^{5}$. 
Tabela 1 - Pacientes portadores de úlcera unilateral de membros inferiores, de acordo com a presença de alteração linfocintilográfica nos membros com úlcera (grupo III: classes 5 e 6) e contralateral sem úlcera (grupos I e II: classes $0,1,2,3$ e 4)

\begin{tabular}{lcccc}
\hline & \multicolumn{2}{c}{ Alteração linfocintilográfica } & & Total \\
\cline { 2 - 3 } Grupo/CEAP & Sim & Não & & \% de sim \\
\hline III & 29 & 11 & 40 & 72,50 \\
I/II & 9 & 31 & 40 & 22,50 \\
Total & 38 & 42 & 80 & 47,50 \\
\hline
\end{tabular}

Qui-quadrado calculado $=20,05 ;$ qui-quadrado crítico $=3,84$. III $>$ I/II $(\mathrm{p}<0,001)$.

A análise qualitativa da linfocintilografia foi feita por observadores independentes, com experiência em medicina nuclear. Os observadores não tinham conhecimento prévio dos pacientes. Para evitar qualquer tendenciosidade na análise dos parâmetros, os exames foram apresentados sem a identificação e história clínica. Os parâmetros analisados bilateralmente foram: presença de linfonodo poplíteo, retenção do radiofármaco, presença de circulação colateral, adenomegalia inguinal e refluxo dérmico. Os dados foram anotados em protocolo específico para cada paciente e submetidos a análise estatística.

Para a análise estatística, foram utilizados testes nãoparamétricos, levando-se em consideração a natureza das variáveis estudadas:

- Teste G de Cochran ${ }^{9}$, para analisar as concomitâncias das várias alterações linfocintilográficas consideradas. Esse teste foi aplicado, separadamente, para os membros inferiores dos três grupos estudados.

- Teste do qui-quadrado para tabelas 2 x 2 ou tabelas 2 x $\mathrm{n}^{9}$, com a finalidade de comparar os grupos com alteração linfocintilográfica e sem alteração linfocintilográfica em relação aos grupos CEAP. A mesma análise foi aplicada para comparar os grupos em relação à presença de linfonodo poplíteo, retenção do radiofármaco, circulação colateral, adenomegalia inguinal e refluxo dérmico. Em todos os testes fixou-se em 0,05 ou $5 \%$ o nível de significância.

\section{Resultados}

Quando foram consideradas as alterações linfocintilográficas em relação à extremidade inferior, comparando o grupo III com os grupos I e II, foi encontrada diferença significativa entre a presença de alteração linfocintilográfica entre os grupos III e os grupos I e II, sendo observadas alterações em 72,50 e em 22,50\%, respectivamente (Tabela 1).

Quando foram comparadas as alterações encontradas na análise linfocintilográfica do grupo III, foram encontradas com maior frequência a presença de refluxo dérmico, adenomegalia inguinal e retenção do radiofármaco e, em menor frequência, a presença de linfonodo poplíteo, sendo tais dados estatisticamente significati$\operatorname{vos}(p<0,001)$. Em relação às linfocintilografias dos grupos I e II, conjuntamente, foi encontrada maior frequência apenas do parâmetro retenção do radiofármaco, sendo esse dado estatisticamente significativo ( $\mathrm{p}$ $<0,010$ ). Quando foram comparadas as alterações entre o grupo III e os grupos I e II conjuntamente, foi encontrada diferença estatisticamente significativa em todos os parâmetros avaliados, exceto na circulação colateral (Tabela 2). Houve concordância entre os observadores $>80 \%$ em todos os parâmetros analisados, sendo que no parâmetro "circulação colateral" ocorreu maior número de divergências, porém sem significância estatística $(\mathrm{p}<0,05 \%)$.

Analisando a presença de alteração linfocintilográfica nos membros inferiores conforme a classificação CEAP, encontramos diferença estatisticamente significante $(\mathrm{p}=0,000)$, sendo a alteração linfocintilográfica mais frequente no grupo III e menos observada nos grupos I e II (Tabela 3).

Quando foram avaliados os membros inferiores de acordo com as alterações linfocintilográficas em relação à avaliação CEAP, observou-se diferença estatisticamente significativa nos parâmetros retenção do 
Tabela 2 - Pacientes portadores de úlcera venosa unilateral, de acordo com as alterações linfocintilográficas nos membros do grupo III (classes 5 e 6 ) e I/II (classes $0,1,2,3$ e 4 )

\begin{tabular}{|c|c|c|c|c|c|c|c|}
\hline \multirow[b]{2}{*}{ Grupos } & \multicolumn{5}{|c|}{ Alterações linfocintilográficas } & \multirow{2}{*}{$\begin{array}{c}\text { Teste G de } \\
\text { Cochran } \\
\text { calculado }\end{array}$} & \multirow[b]{2}{*}{$\mathbf{p}$} \\
\hline & $\begin{array}{c}\text { Linfonodo } \\
\text { poplíteo }\end{array}$ & $\begin{array}{l}\text { Refluxo } \\
\text { dérmico }\end{array}$ & $\begin{array}{c}\text { Circulação } \\
\text { colateral }\end{array}$ & $\begin{array}{c}\text { Adenomegalia } \\
\text { inguinal }\end{array}$ & $\begin{array}{r}\text { Retenção do } \\
\text { radiofármaco }\end{array}$ & & \\
\hline III $(n=40)$ & $7 *$ & $19^{\dagger}$ & $11^{*}$ & $13^{\S}$ & $27^{\|}$ & 33,32 & $<0,001$ \\
\hline Porcentagem & 17,5 & 47,5 & 27,5 & 32,5 & 67,5 & & \\
\hline $\mathrm{I} / \mathrm{II}(\mathrm{n}=40)$ & $1^{*}$ & $3^{\dagger}$ & $6^{*}$ & $4^{\S}$ & $11^{\| \prime}$ & 17,06 & $<0,010$ \\
\hline Porcentagem & 2,5 & 7,5 & 15,0 & 10,0 & 27,5 & & \\
\hline
\end{tabular}

$\chi^{2}=$ qui-quadrado.

$*$ Teste t de Fisher, $\mathrm{p}=0,0283$, III $>$ I/II.

$\chi^{2}=16,05, \mathrm{p}<0,0001$, III $>$ I/II.

$\ddagger \chi^{2}=1,87, \mathrm{p}>0,05$ (não-significante).

$\S \chi^{2}=6,05, \mathrm{p}<0,05$, III $>$ I/II.

"I $\chi^{2}=12,83, \mathrm{p}<0,001$, III $>$ I/II.

Tabela 3 - Distribuição dos membros (80) de acordo com a presença de alteração linfocintilográfica e a classificação clínica nos grupos I, II e III - CEAP (classes 0 a 6 )

\begin{tabular}{lcccc}
\hline \multirow{2}{*}{ Grupos } & \multicolumn{2}{c}{ Alterações linfocintilográficas } & & Total \\
\cline { 2 - 3 } III & Presente & Ausente & & \% de presença \\
II & 29 & 11 & 40 & 72,5 \\
I & 8 & 18 & 26 & 30,8 \\
Total & 1 & 13 & 14 & 7,1 \\
\hline
\end{tabular}

Qui-quadrado $=22,09(\mathrm{p}<0,001)$.

III > II e I.

II $>$ I.

radiofármaco (Figuras 1, 2, 3 e 4), adenomegalia inguinal (Figura 2) e refluxo dérmico (Figuras 1 e 4). Não foi encontrada diferença estatisticamente significativa para os parâmetros linfonodo poplíteo (Figura 2) e circulação colateral (Figura 3) (Tabela 4).

\section{Discussão}

A influência das doenças venosas no funcionamento do sistema linfático tem sido pouco avaliada, embora possa haver implicações fisiopatológicas e terapêuticas. Tanto as doenças venosas quanto as linfáticas podem manifestar-se pelo edema, e o diagnóstico diferencial nem sempre é simples, principalmente na fase inicial dos linfedemas. Há relatos na literatura de que membros inferiores com hipertensão venosa crônica têm um fluxo linfático aumentado e que, de acordo com a linfocintilografia, esse aumento pode chegar duas a três vezes acima do normal ${ }^{10}$.

A literatura é rica em trabalhos publicados sobre a acurácia da avaliação do sistema linfático pela linfocintilografia para diagnóstico, sendo considerado o método inicial de escolha para afastar ou confirmar lesões linfáticas, já que, através da avaliação qualitativa, temos sensibilidade variando de 70 a $92 \%$ e especificidade de $100 \%{ }^{4,5}$. É um exame objetivo, seguro e rápido, detectando alterações mais precocemente e com poucas complicações; além disso, tem baixa radiação e oferece 


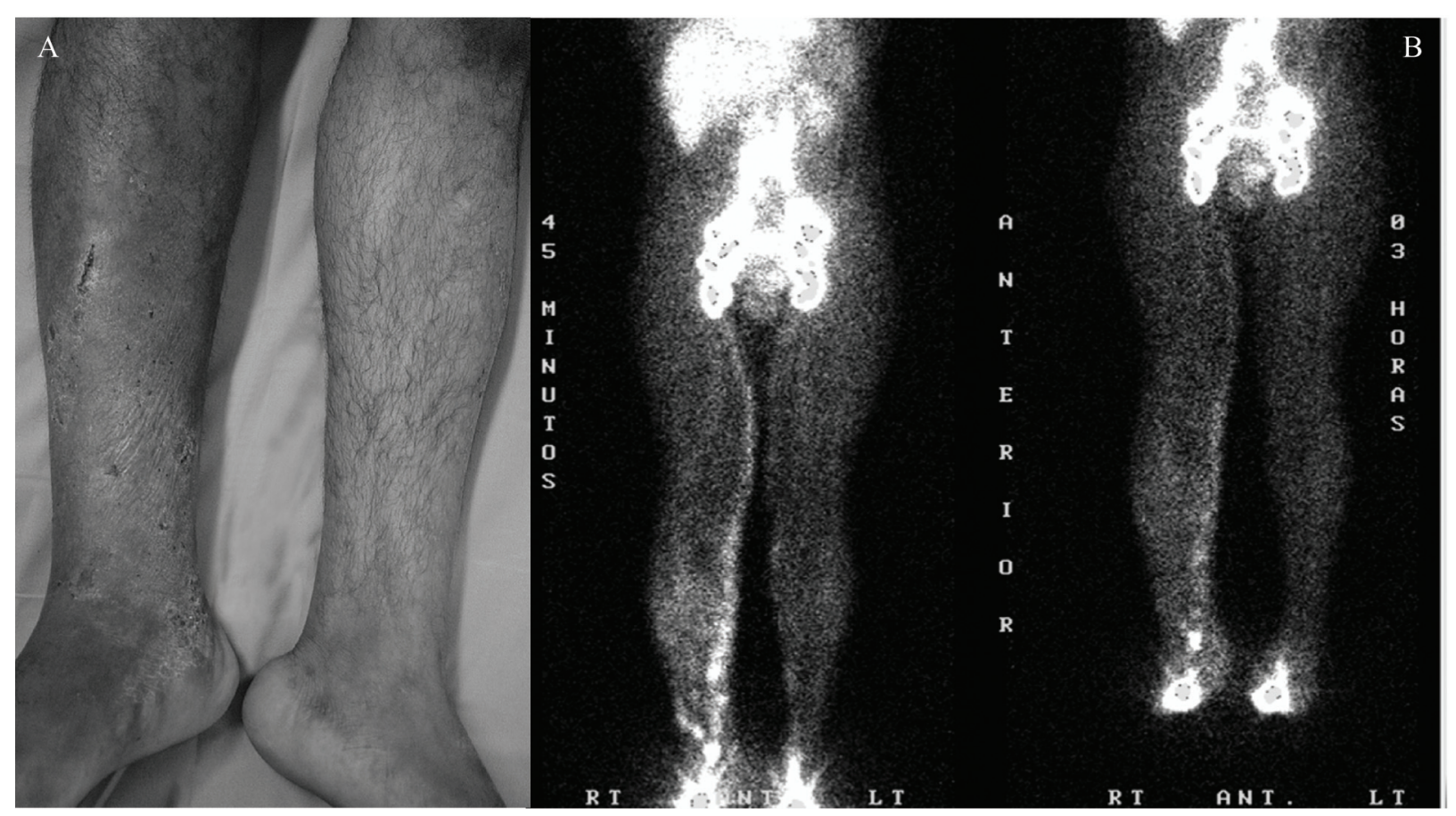

Figura 1 - A) Paciente masculino, 42 anos, úlcera de membro inferior direito há 18 meses, com 2 recorrências; B) linfocintilografia com refluxo dérmico e retenção do radiofármaco no membro inferior direito. Membro inferior esquerdo com aspecto normal

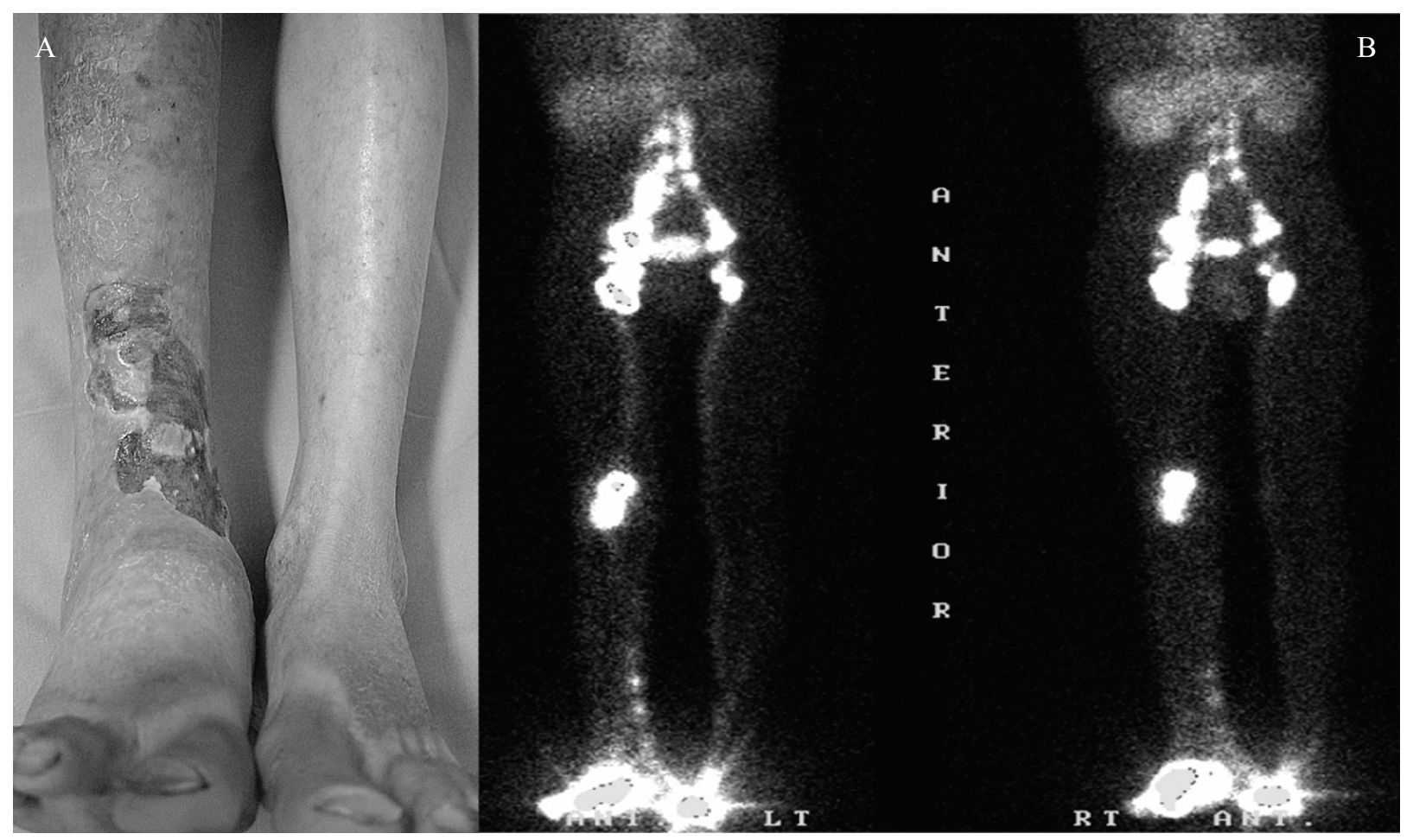

Figura 2 - A) Paciente masculino, 66 anos, apresentando úlcera em membro inferior direito com duração de 120 meses e 3 recorrências; B) linfocintilografia evidenciando linfonodo poplíteo, adenomegalia inguinal e retenção de contraste no local da úlcera à direita. Membro inferior esquerdo com aspecto normal 


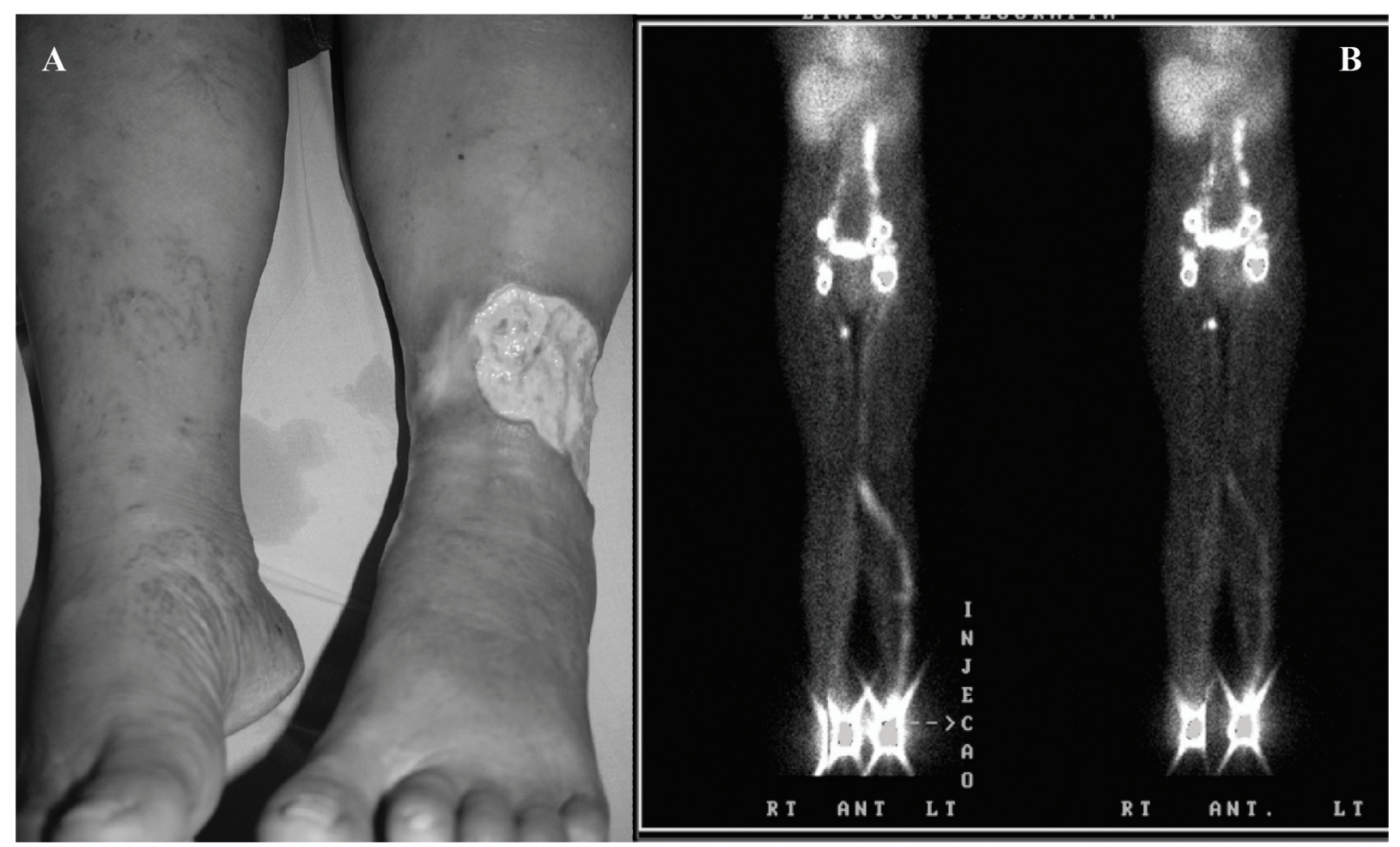

Figura 3 - A) Paciente feminina, 47 anos, com úlcera no membro inferior esquerdo, com 60 meses de duração; B) linfocintilografia mostra presença de circulação colateral na perna, adenomegalia inguinal, retardo de radiofármaco. A direita mostra linfonodo em coxa

imagens identificáveis das alterações anatômicas e funcionais dos linfáticos e linfonodos, sendo facilmente repetido, se necessário ${ }^{5,11-15}$. Foi utilizado o Dextran tecnécio $^{99 \mathrm{~m}}$, pela facilidade de obtenção do material e de armazenamento dos dados e pela qualidade satisfatória das imagens, como já padronizado e publicado em estudos anteriores ${ }^{5,12-14,16-19}$. Os itens analisados foram: presença de linfonodo poplíteo, que pode ser considerado sinal da falência da drenagem pelas vias linfáticas normais; refluxo dérmico, por extravasamento do radioisótopo pela hipertensão nos coletores linfáticos próximos à lesão ulcerada e/ou cicatrizada; adenomegalia inguinal, em decorrência, provavelmente, das infecções de repetição da úlcera; retenção do radiofármaco, por sobrecarga dos coletores linfáticos pelo aumento da pressão hidrostática e presença de circulação colateral. A circulação colateral é formada por uma rede de comunicações não-funcionais no estado fisiológico, podendo transformar-se em vias de suplência, quando as correntes linfáticas de escoamento principais estão saturadas ou bloqueadas, sendo consideradas vias não-habituais ${ }^{20}$.
Em relação às principais alterações linfocintilográficas encontradas nos membros com úlcera (linfonodo poplíteo, retenção do radiofármaco, refluxo dérmico e adenomegalia inguinal) e nos membros sem úlcera (retenção do radiofármaco), este estudo encontrou resultados semelhantes aos relatados por outros autores $^{21,22}$, que demonstraram alterações morfológicas nos linfáticos superficiais nas áreas de dermatoesclerose, sugerindo que as infecções locais e fibrose periúlcera causam lesão dos linfáticos superficiais, levando ao refluxo dérmico e retenção do radiofármaco.

Outro estudo da função linfática, avaliada através de análise quantitativa, observou que a função linfática estava diminuída nas pernas ulceradas em comparação com as normais, além de drenagem linfática diminuída com a idade nos indivíduos controles, acima de $65 \mathrm{anos}^{7}$, supondo que o aumento da idade deteriora a função intrínseca da bomba linfática e a capacidade de transporte do sistema linfático ${ }^{23}$.

Vários autores descreveram alterações em linfografias diretas realizadas em pacientes portadores de trombose venosa profunda, doença varicosa dos membros 


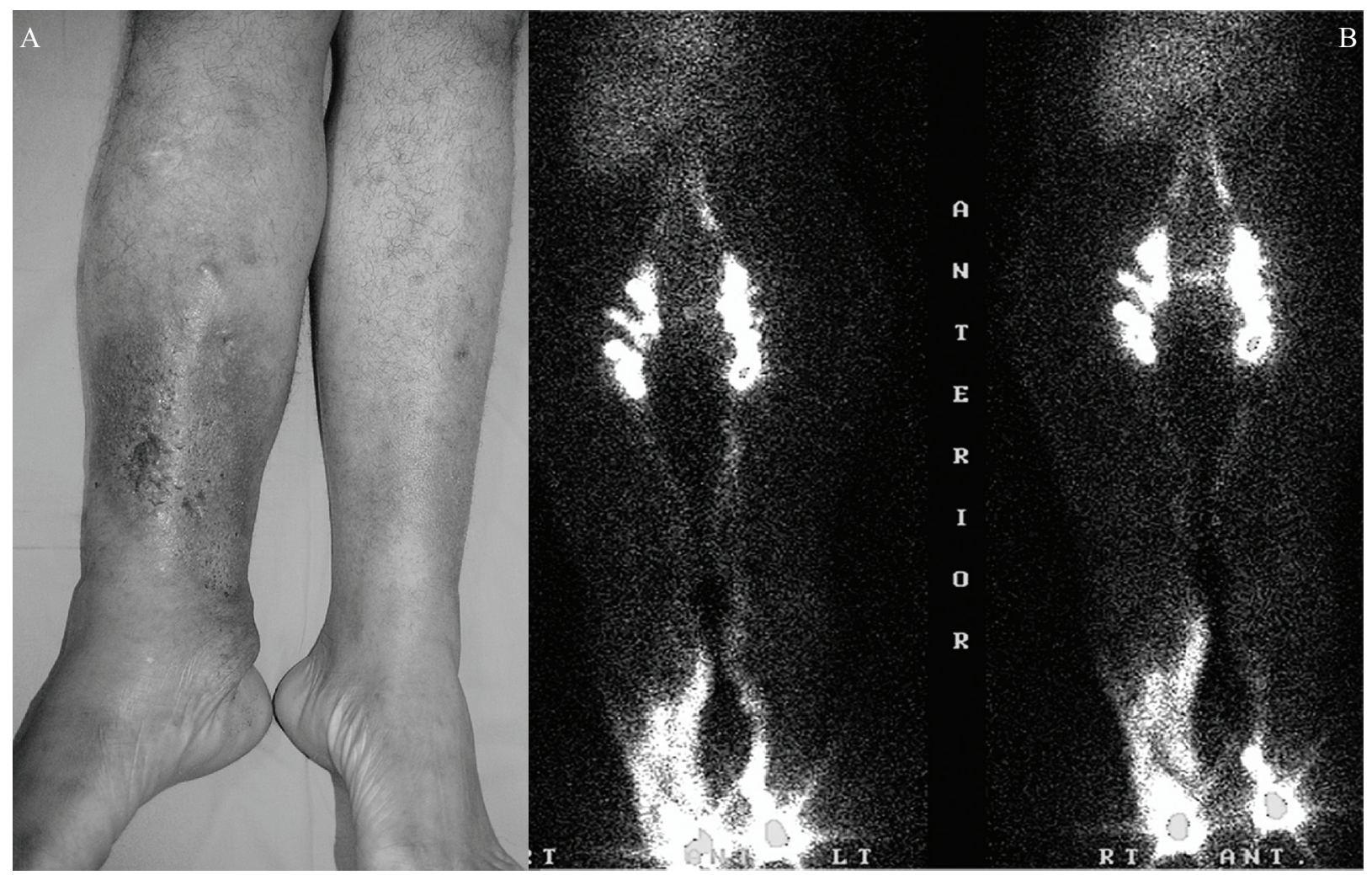

Figura 4 - A) Paciente masculino, 28 anos, úlcera em membro inferior direito com duração de 24 meses e 1 recorrência; B) linfocintilografia evidenciando refluxo dérmico à direita e retenção do radiofármaco bilateral

inferiores com edema crônico e síndrome póstrombótica, mostrando vasos linfáticos pouco mais calibrosos, dilatados e tortuosos (contas de rosário) e linfonodos mais e precocemente contrastados em relação ao membro normal ${ }^{24-26}$. Em pacientes com úlceras secundárias à hipertensão venosa crônica, também descreveram dilatações e tortuosidades de vasos coletores, extravasamento de contraste na região da úlcera e fluxo de contraste pela derme, sem a opacificação de vasos coletores. $\mathrm{O}$ aspecto era compatível com diagnóstico de linfedema e similar ao das linfocintilografias ${ }^{27}$, em relação ao refluxo dérmico na extremidade e à concentração do radiofármaco no local da úlcera, também observado no presente estudo.

Utilizando a linfocintilografia em pacientes com edema decorrente de obstruções venosas (tromboses venosas femorais ou poplíteas), insuficiência cardíaca congestiva e hipoproteinemia, foi observado aumento na velocidade de remoção do radiofármaco ${ }^{28}$. Outros estudos não mostram padrões uniformes de fluxo linfático $^{29}$, sendo encontrados: fluxo mais acelerado da linfa quanto mais precoce o diagnóstico da doença venosa ${ }^{30}$; redução do radiofármaco, semelhantemente aos encontrados em pacientes com linfedemas primários ${ }^{31}$; destruição linfática extensa na região acometida e consequente redução do fluxo linfático ${ }^{32}$; e transporte linfático diminuído nos linfáticos profundos e aumentado nos superficiais na fase precoce ${ }^{33}$, porém diminuído quando havia úlcera de estase ou celulites e/ou linfangites recorrentes ${ }^{6}$. Com a progressão da doença e suas complicações (ulcerações recorrentes de pele), o fluxo linfático e consequente transporte de linfa decres$\mathrm{ce}^{3,34}$, encontrando-se lenteado em pacientes com insuficiência venosa. Tal resultado contraria estudo prévio, quando foi verificado um aumento do fluxo inguinal nos membros com edema de origem venosa, comparado aos membros normais ${ }^{10}$. Essa diferença no transporte linfático da insuficiência venosa pode ser explicada pela duração do edema. Na trombose venosa aguda ou no início da insuficiência venosa crônica, tem-se um aumento na filtração capilar e um transporte mais rápido nos canais linfáticos normais ${ }^{35}$. Posteriormente, com a constante 
Tabela 4 - Distribuição dos membros de acordo com as alterações linfocintilográficas e a avaliação clínica CEAP (classes 0 a 6 grupos I,II e III) (resultado do teste do qui-quadrado)

\begin{tabular}{|c|c|c|c|c|c|c|c|c|}
\hline \multirow{3}{*}{$\begin{array}{l}\text { Alterações } \\
\text { linfocintilográficas }\end{array}$} & \multicolumn{6}{|c|}{ Clínica } & \multirow{3}{*}{$\begin{array}{c}\chi^{2} \\
\text { calculado }\end{array}$} & \multirow[b]{3}{*}{$\mathbf{p}$} \\
\hline & \multicolumn{2}{|c|}{$\begin{array}{l}\text { Classes } 5 \text { e } 6 \\
\text { (grupo III) }\end{array}$} & \multicolumn{2}{|c|}{$\begin{array}{c}\text { Classes 2, } 3 \text { e } 4 \\
\text { (grupo II) }\end{array}$} & \multicolumn{2}{|c|}{$\begin{array}{c}\text { Classes } 0 \text { e } 1 \\
\text { (grupo I) }\end{array}$} & & \\
\hline & $\mathbf{n}$ & $\%$ & $\mathbf{n}$ & $\%$ & $\mathbf{n}$ & $\%$ & & \\
\hline \multicolumn{9}{|l|}{ Linfonodo poplíteo } \\
\hline Presente & 7 & 17,5 & 1 & 3,8 & 0 & 0,0 & 5,15 & $<0,05$ \\
\hline Ausente & 33 & 82,5 & 25 & 96,2 & 14 & 100,0 & & \\
\hline Total & 40 & 100,0 & 26 & 100,0 & 14 & 100,0 & & \\
\hline \multicolumn{9}{|l|}{$\begin{array}{l}\text { Retenção do } \\
\text { radiofármaco }\end{array}$} \\
\hline Presente & 27 & 67,5 & 9 & 34,6 & 1 & 7,1 & 17,30 & $<0,001$ \\
\hline Ausente & 13 & 32,5 & 17 & 65,4 & 13 & 92,9 & & \\
\hline Total & 40 & 100,0 & 26 & 100,0 & 14 & 100,0 & & \\
\hline \multicolumn{9}{|l|}{ Circulação colateral } \\
\hline Presente & 11 & 27,5 & 5 & 19,2 & 1 & 7,1 & 2,66 & $<0,05$ \\
\hline Ausente & 29 & 72,5 & 21 & 80,8 & 13 & 92,9 & & \\
\hline Total & 40 & 100,0 & 26 & 100,0 & 14 & 100,0 & & \\
\hline \multicolumn{9}{|l|}{$\begin{array}{l}\text { Adenomegalia } \\
\text { inguinal }\end{array}$} \\
\hline Presente & 13 & 32,5 & 2 & 7,7 & 1 & 7,1 & 7,81 & $<0,01$ \\
\hline Ausente & 27 & 67,5 & 24 & 92,3 & 13 & 92,9 & & \\
\hline Total & 40 & 100,0 & 26 & 100,0 & 14 & 100,0 & & \\
\hline \multicolumn{9}{|l|}{ Refluxo dérmico } \\
\hline Presente & 19 & 47,5 & 2 & 7,7 & 1 & 7,1 & 16,05 & $<0,001$ \\
\hline Ausente & 21 & 52,5 & 24 & 92,3 & 13 & 92,9 & & \\
\hline Total & 14 & 100,0 & 26 & 100,0 & 40 & 100,0 & & \\
\hline
\end{tabular}

lesão dos capilares linfáticos no subcutâneo pela infecção e fibrose local, a capacidade de transporte de linfa fica comprometida, instalando-se o edema no membro ${ }^{3,32}$.

Não foram encontrados na literatura estudos avaliando qualitativamente as alterações linfocintilográficas em pacientes com úlceras de estase unilateral, classificados de acordo com a CEAP, para comparação com os resultados do presente estudo. Conclui-se, portanto, que as alterações linfocintilográficas mais frequentes encontradas neste estudo, nos membros com úlcera, são em decorrência da própria lesão da pele e pelo tempo de estase venosa crônica. Com base nas evidências disponíveis, a hipótese é a de que os canais linfáticos não desempenham uma função importante no início da estase venosa, mas são afetados pela sobrecarga, levando às alterações dos capilares e do líquido intersticial e, a partir daí, contribuem como fator secundário para a deterioração da pele e do tecido subcutâneo. A lesão linfática é um importante componente na persistência da úlcera venosa crônica e pode explicar a dificuldade no tratamento eficaz das úlceras complicadas das pernas. 


\section{Referências}

1. Browse NL, Burnand KG, Irvine AT, Wilson NM. Insuficiência venosa crônica. In: Browse NL, Burnand KG, Irvine AT, Wilson NM. Doenças venosas. $2^{\mathrm{a}}$ ed. Rio de Janeiro: DiLivros; 2001.

2. Nicolaides AN, Cardiovascular Disease Educational and Research Trust, European Society of Vascular Surgery, et al. Investigation of chronic venous insufficiency. A consensus statement. (France, March 5-9, 1997). Circulation. 2000;102:E126-63.

3. Bräutigam P, Vanscheidt W, Földi E, Krause T, Moser E. The importance of the subfascial lymphatic in the diagnosis of lower limb edema: investigations with semiquantitative lymphoscintigraphy. Angiology. 1993;44:464-70.

4. Gloviczki P, Calcagno D, Schirger A, et al. Noninvasive evaluation of the swollen extremity: experiences with 190 lymphoscintigraphic examinations. J Vasc Surg. 1989;9:683-9.

5. Perez MCJ. Linfocintilografia radioisotópica, tomografia computadorizada e ressonância magnética nas doenças linfáticas. In: Maffei FHA. Doenças vasculares periféricas. $3^{\mathrm{a}}$ ed. São Paulo: MEDSI; 2002. p. 545-52.

6. Mosbeck A, Partsch H. Examens de lymphographie isotopique dans le syndrome post-thrombotique. Phebologie. 1991;44:227-35.

7. Bull RH, Gane JN, Evans JEC, Joseph AE, Mortimer PS. Abnormal lymph drainage in patients with chronic venous leg ulcers. J Am Acad Dermatol. 1993;28:585-90.

8. Eklöf B, Rutherford RB, Bergan JJ, et al. Revision of the CEAP classification for chronic venous disorders: consensus statement. J Vasc Surg. 2004;40:1248-52.

9. Siegel S, Castellan Jr. NJ. Nonparametric statistics. 2nd ed. New York: McGraw-Hill; 1988. p. 399.

10. Stewart G, Gaunt JI, Croft DN, Browse NL. Isotope lymphography: a new method of investigating the role of lymphatics in chronic limb oedema. Br J Surg. 1985;72:906-9.

11. Ter SE, Alavi A, Kim CK, Merli G. Lymphoscintigraphy. A reliable test for the diagnosis of lymphedema. Clin Nucl Med. 1993;18:646-54.

12. Miranda F Jr, Perez MC, Castiglioni ML, et al. Effect of sequential intermittent pneumatic compression on both leg lymphedema volume and on lymph transport as semiquantitatively evaluated by lymphoscintigraphy. Lymphology. 2001;34:135-41.

13. Kafejian-Haddad AP, Perez JM, Castiglioni ML, Miranda Junior F, de Figueiredo LF. Lymphscintigraphic evaluation of manual lymphatic drainage for lower extremity lymphedema. Lymphology. 2006;39:41-8.

14. Gomes SC. Linfocintilografia superficial de membros inferiores com dextran $500 \mathrm{Tc} 99 \mathrm{~m}$. Estudo em pacientes portadores de filariose e de linfopatia não filariótica [tese]. Recife: Universidade Federal de Pernambuco; 1990.

15. Guedes-Neto HJ. Estudo linfocintilográfico qualitativo dos membros superiores de doentes com linfedema secundário a tratamento cirúrgico para o câncer de mama [tese]. São Paulo: Faculdade de Ciências Médicas da Santa Casa de São Paulo; 2002.
16. Henze E, Robinson GD, Kuhl DE, Schelbert HR. Tc-99m dextran: a new blood pool-labeling agent for radionuclide angiocardiography. J Nucl Med. 1982;23:348-53.

17. Henze E, Schelbert HR, Collins JD, Najafi A, Bennet LR. Lymphoscintigraphy with Tc-99m labeled dextran. J Nucl Med. 1982;23:923-9.

18. Cestari SC. Contribuição ao estudo dos linfedemas dos membros inferiores com o emprego da linfocintilografia [tese]. São Paulo: Universidade Federal de São Paulo - Escola Paulista de Medicina; 1992.

19. Cestari SC, Petri V, Castiglioni ML, Lederman H. Linfedemas dos membros inferiores: estudo linfocintilográfico. Rev Assoc Med Bras. 1994;40:93-100.

20. Andrade MF, Jacomo AL. Anatomia médico-cirúrgica do sistema linfático dos membros. In: Maffei FH. Doenças vasculares periféricas. $3^{\text {a }}$ ed. São Paulo: MEDSI; 2002. p.169-78.

21. Bollinger A, Isenring G, Franzeck UK. Lymphatic microangiopathy: a complication of severe chronic venous incompetence (CVI). Lymphology. 1982;15:60-5.

22. Bollinger A, Pfister G, Hoffmann U, Franzeck UK. Fluorescence microlymphography in chronic venous incompetence. Int Angiol. 1989;8(4suppl): 23-6.

23. Suga K, Kume N, Matsunaga N, Motoyama K, Hara A, Ogasawara N. Assessment of leg oedema by dynamic lymphoscintigraphy with intradermal injection of technetium-99m human serum albumin and load produced by standing. Eur j Nucl Med. 2001;28:294-303.

24. Kinmonth JB. The lymphatics. Diseases, lymphography and surgery. London: Edward-Arnold; 1972.

25. Crockett DJ. Lymphatic anatomy and lymphoedema. Br J Plast Surg. 1965;18:12-25.

26. Bower R, Danese C, Debbas J, Howard JM. Advances in diagnosis of diseases of the lymphatics. JAMA. 1962;25:687-91.

27. Meade JW, Mueller CB. Lymphatic and venous examination of the ulcerated leg: a preliminary report. Surgery. 1992;112:872-5.

28. Hollander W, Reilly P, Burrows BA. Lymphatic flow in human subjects as indicated by the disappearance of 131I-labeled albumin from the subcutaneous tissue. J Clin Invest. 1961;40:222-33.

29. Rijke AM, Croft BY, Johnson RA, de Jongste AB, Camps JA. Lymphoscintigraphy and lymphedema of the lower extremities. J Nucl Med. 1990;31:990-8.

30. Cambria RA, Gloviczski P, Naessens JM, Wahner HW. Noninvasive evaluation of the lymphatic system with lymphoscintigraphy: a prospective, semi-quantitative analysis in 386 extremities. J Vasc Surg. 1993;18:773-82.

31. Weissleder H, Weissleder R. Lymphedema: evaluation of quantitative lymphoscinthigraphy in 238 patients. Radiology. 1988;167:729-35.

32. Larcos G, Wahner HW. Lymphoscintigraphic abnormalities in venous thrombosis. J Nucl Med. 1991;32:2144-8. 
33. Bräutigam P, Földi E, Schaiper I, Krause T, Vanscheidt W, Moser E. Analysis of lymphatic drainage in various forms of leg edema using two compartment lymphoscintigraphy. Lymphology. 1998;31:43-55.

34. Collins PS, Villavicencio JL, Abreu SH, et al. Abnormalities of lymphatic drainage in lower extremities: a lymphoscintilographic study. J Vasc Surg. 1989;9:145-52.

35. Andrade MFC. Avaliação da absorção e transporte linfáticos em pacientes com trombose venosa profunda de membros inferiores através da linfocintilografia [tese]. São Paulo: Universidade de São Paulo; 1998.
Correspondência:

Maria del Carmen Janeiro Perez

Rua Borges Lagoa, 710/14, Vila Clementino CEP 04038-031 - São Paulo, SP

Tel.: (11) 5575.6085

Fax: (11) 5575.6085

\section{Submissão de figuras ao J Vasc Bras}

\section{Prezados Autores:}

Estamos numa campanha para melhorar a qualidade das imagens publicadas no Jornal Vascular Brasileiro.

Contribua você também: leia as normas e certifique-se de enviar figuras que atendam aos requisitos técnicos e de qualidade.

Figuras devem ser enviadas em formato eletrônico (exclusivamente gráficos e fotografias digitais), em arquivos independentes, nas extensões .jpg, gif ou .tif, com resolução mínima de 300 dpi. Quando não for possível enviar as figuras por e-mail, o envio deve ser feito via correio. 